\title{
Analysis of an Epidemic Spreading Model with Exponential Decay Law
}

\author{
Mehmet Yavuz* and Necati Özdemir
}

\begin{abstract}
Mathematical modeling of infectious diseases has shown that combinations of isolation, quarantine, vaccine, and treatment are often necessary in order to eliminate most infectious diseases. Continuous mathematical models have been used to study the dynamics of infectious diseases within a human host and in the population. We have used in this study a SIR model that categorizes individuals in a population as susceptible (S), infected (I) and recovered (R). It also simulates the transmission dynamics of diseases where individuals acquire permanent immunity. We have considered the SIR model using the Caputo-Fabrizio and we have obtained special solutions and numerical simulations using an iterative scheme with Laplace transform. Moreover, we have studied the uniqueness and existence of the solutions.
\end{abstract}

Keywords: Epidemic model; exponential law; numerical simulation; fractional derivative; non-singularity.

AMS Subject Classification (2020): Primary: 26A33; 37C25; Secondary: 92D30.

${ }^{*}$ Corresponding author

\section{Introduction}

Infectious diseases that confer permanent immunity are modelled by the epidemiological models [1]. One of the most important epidemiological models which are used extensively is the SIR model for the spread of disease, which consists of a three-dimensional system that describes the changes in the number of susceptible, infected, and recovered individuals in a given population. This model was firstly developed by Kermack and McKendrick [2] in 1927, and despite its simplicity, it is a useful and applicable model for many infectious diseases. Many researchers have done interesting studies about the SIR model and its different special modifications in the literature. For instance, Arqub and El-Ajou [3] have obtained a solution for the fractional SIR model by using homotopy analysis method (HAM). They have used the Caputo fractional derivative operator in their study. El-Saka [4] has used the fractional order SIR and SIRS models in order to model an infectious disease with variable population size by using the Caputo derivative operator. In another study, Angstmann et al. [5] have derived a fractional-order infectivity SIR model from a stochastic process that incorporates a time-since-infection dependence on the infectivity of individuals. Arenas et al. [6] have developed a nonstandard finite difference (NSFD) method to obtain numerical solutions of the fractional SI and SIR epidemic models by using Caputo and Grünwald-Letnikov fractional operators.

Angstmann et al. [7] have constructed an SIR model for an epidemic, including vital dynamics, from an underlying stochastic process. They have concluded that how fractional differential operators arise naturally in epidemiological models whenever the recovery time from the disease is power-law distributed. Demirci et al [8] have introduced a fractional SEIR epidemic model with vertical transmission, where the death rate of the population is density dependent. They have also assumed that there exists an infection related death rate. They have showed the existence of non-negative solutions of the model, and also give a detailed stability analysis of disease free and positive fixed points. Finally, they have supported their theoretical aspects with a numerical example. In addition to these studies, in the literature there are many papers demonstrate to model other epidemic models and special diseases such as modelling the spread of computer virus [9], stability analysis of a fractional

Received : 15-01-2019, Accepted : 22-11-2019 
human African trypanosomiasis model [10], a schistosomiasis disease model [11], a novel framework for blood vessels detection in retinal images [12], a parabolic fractional degenerate problem emerging in a spatial diffusion of biological population model [13], SIRI epidemic model with distributed delay and relapse [14], etc.

On the other hand, in the recent years, some useful and effective fractional operators with non-singular kernel have been investigated. In this sense, Caputo and Fabrizio [15] firstly have defined a derivative operator of fractional order which is called as Caputo-Fabrizio (CF) fractional derivative based on exponential law which meaning in many natural processes and also has crossover effect with statistical representation. Then, Atangana and Baleanu [16] have introduced a new definition which is called as Atangana-Baleanu (AB) in terms of the Mittag-Leffler function. The $\mathrm{AB}$ operator has also been regarded as a filter with fractional regulator and it has the fundamental properties of usual fractional derivative and integrals [17]. These operators have shown to be the future for modeling of several scientific processes including chaotic theory, control theory, financial problem. Moreover, the Laplace transform of the $\mathrm{CF}$ and $\mathrm{AB}$ operators require the physically interpretable initial conditions with integer order derivatives and so it is rather preferred for modelling of different physical process. The two operators have strong statistical representation which can be interpreted in real-life sense. That is why their considerable properties, they have an increasing interest in different real-life problems [19-48]. In 2017, Yang et al. [18] have defined a new fractional operator based on the normalized sinc function (NSF). These fractional operators also have a nonlocal kernel. Therefore, the non-locality of the kernel gives opportunity to have better description of memory properties in the structures with different scales.

In this study, the main reason for regarding as the operator is fractional instead of integer order is that the integer order system can be considered as a special case from the fractional order system by putting the time-fractional derivative equal to unity. Moreover, it is a fact that using differential equations of fractional order (FDEs) can help us to reduce the errors deriving from the unheeded parameters in modelling of real life problems $[3,49,50]$. Also, FDEs are innately reference to systems with memory, which holds in most biological systems. Since, the study found that fractional derivative was very suitable to describe long memory and hereditary properties of various materials and processes [51].

\section{Some Preliminaries}

Let us remind some well-known mathematical tools needed to use in the present study. The Caputo-Fabrizio fractional derivative in Caputo sense (CFC) is defined by [15]

$$
{ }_{0}^{C F C} D_{\tau}^{\varepsilon} \zeta(\tau)=\frac{\kappa(\varepsilon)}{1-\varepsilon} \int_{0}^{\tau} \zeta^{\prime}(m) \exp \left[-\frac{\varepsilon(\tau-m)}{1-\varepsilon}\right] d m, \quad 0<\varepsilon \leq 1
$$

where $\kappa(\varepsilon)$ is a normalization function that equals to 1 when $\varepsilon=0$ and $\varepsilon=1$. In the present study, we apply the Laplace transform of CF derivative according to time variable $\tau$. Considering the convolution property of Laplace transform (LT), we have the following transformation of ${ }_{0}^{C F C} D_{\tau}^{\varepsilon} \zeta(\tau)[15,52]$ :

$$
\mathcal{L}\left\{{ }_{0}^{C F C} D_{\tau}^{\varepsilon} \zeta(\tau)\right\}(\ell)=\frac{\ell \mathcal{L}\{\zeta(\tau)\}-\zeta(0)}{\ell+\varepsilon(1-\ell)} .
$$

In addition, the integral definition of order $\varepsilon, \quad(0<\varepsilon \leq 1)$ of the function $\zeta(\tau)$ is given by [53]

$$
{ }_{0}^{C F} \mathrm{I}_{\tau}^{\varepsilon} \zeta(\tau)=\frac{2(1-\varepsilon)}{(2-\varepsilon) \kappa(\varepsilon)} \zeta(\tau)+\frac{2 \varepsilon}{(2-\varepsilon) \kappa(\varepsilon)} \int_{0}^{\tau} \zeta(s) d s, \quad \tau \geq 0
$$

where

$$
\kappa(\varepsilon)=\frac{2}{2-\varepsilon}, \quad 0<\varepsilon<1 .
$$

\section{SIR Epidemic Model with Exponential Decay Law}

In this work, we consider a population that has three individuals groups: susceptible, infected and recovered. Their dynamics are modelled by standard SIR problem [54] and throughout the study, we demonstrate these individuals with the notations $\xi_{1}, \xi_{2}, \xi_{3}$, respectively. We show the mentioned epidemic model with the integerorder as

$$
\begin{aligned}
& \xi_{1}^{\prime}(\tau)=\lambda-\left(\alpha \xi_{2}(\tau)+\lambda\right) \xi_{1}(\tau), \\
& \xi_{2}^{\prime}(\tau)=\alpha \xi_{1}(\tau) \xi_{2}(\tau)-(\lambda+\delta) \xi_{2}(\tau), \\
& \xi_{3}^{\prime}(\tau)=\delta \xi_{2}(\tau)-\lambda \xi_{3}(\tau)
\end{aligned}
$$


where $\lambda, \alpha$ and $\delta$ are system parameters. In the model 3.1, $\xi_{1}(\tau)$ represents the rate of individuals susceptible to the illness, who are born and die at the same proportion $\lambda$. Therefore their life expectancy average is $1 / \lambda$. Susceptible becomes infected at the proportion $\alpha \xi_{2}(\tau)$, where $\xi_{2}(\tau)$ is the rate of infected individuals and $\alpha$ is the communication rate. Infected individuals become recovered (i.e. acquire long life immunity) at a rate $\delta>0$, so that $1 / \delta$ is their infectious process average. Infected individuals who have recovered are represented by the proportion $\xi_{3}$.

Considering the SIR model in Eq. 3.1, the generalized SIR epidemic model with a derivative operator which is defined by exponential function is presented by

$$
\begin{aligned}
& { }_{0}^{C F C} D_{\tau}^{\varepsilon} \xi_{1}(\tau)=\lambda-\left(\alpha \xi_{2}(\tau)+\lambda\right) \xi_{1}(\tau), \\
& { }_{C}^{C} F C D_{\tau}^{\varepsilon} \xi_{2}(\tau)=\alpha \xi_{1}(\tau) \xi_{2}(\tau)-(\lambda+\delta) \xi_{2}(\tau), \\
& { }_{0}^{C F C} D_{\tau}^{\varepsilon} \xi_{3}(\tau)=\delta \xi_{2}(\tau)-\lambda \xi_{3}(\tau),
\end{aligned}
$$

where ${ }_{0}^{C F C} D_{\tau}^{\varepsilon}$ demonstrates the Caputo-Fabrizio fractional operator of order $0<\varepsilon \leq 1$. This mentioned fractional epidemic model has the following initial conditions

$$
\xi_{1(0)}(\tau)=\xi_{1}(0) ; \quad \xi_{2(0)}(\tau)=\xi_{2}(0) ; \quad \xi_{3(0)}(\tau)=\xi_{3}(0) .
$$

By taking into account the fixed-point theorem, we determine the existence of the solution of the system 3.2. We rewrite Eq. 3.2 in terms of an integral equation by using the integral operator mentioned in Eq. 2.3 as the following:

$$
\begin{aligned}
& \xi_{1}(\tau)-\xi_{1}(0)={ }_{0}^{C F} \mathrm{I}_{\tau}^{\varepsilon}\left[\lambda-\left(\alpha \xi_{2}(\tau)+\lambda\right) \xi_{1}(\tau)\right], \\
& \xi_{2}(\tau)-\xi_{2}(0)={ }_{0}^{C F} \mathrm{I}_{\tau}^{\varepsilon}\left[\alpha \xi_{1}(\tau) \xi_{2}(\tau)-(\lambda+\delta) \xi_{2}(\tau)\right], \\
& \xi_{3}(\tau)-\xi_{3}(0)={ }_{0}^{C F} \mathrm{I}_{\tau}^{\varepsilon}\left[\delta \xi_{2}(\tau)-\lambda \xi_{3}(\tau)\right] .
\end{aligned}
$$

Applying the integral operator we have the following system

$$
\begin{aligned}
\xi_{1}(\tau)= & \xi_{1}(0)+\frac{2(1-\varepsilon)}{(2-\varepsilon) \kappa(\varepsilon)}\left[\lambda-\left(\alpha \xi_{2}(\tau)+\lambda\right) \xi_{1}(\tau)\right] \\
& +\frac{2 \varepsilon}{(2-\varepsilon) \kappa(\varepsilon)} \int_{0}^{\tau}\left[\lambda-\left(\alpha \xi_{2}(k)+\lambda\right) \xi_{1}(k)\right] d k, \\
\xi_{2}(\tau)= & \xi_{2}(0)+\frac{2(1-\varepsilon)}{(2-\varepsilon) \kappa(\varepsilon)}\left[\alpha \xi_{1}(\tau) \xi_{2}(\tau)-(\lambda+\delta) \xi_{2}(\tau)\right] \\
& +\frac{2 \varepsilon}{(2-\varepsilon) \kappa(\varepsilon)} \int_{0}^{\tau}\left[\alpha \xi_{1}(k) \xi_{2}(k)-(\lambda+\delta) \xi_{2}(k)\right] d k, \\
\xi_{3}(\tau)= & \xi_{3}(0)+\frac{2(1-\varepsilon)}{(2-\varepsilon) \kappa(\varepsilon)}\left[\delta \xi_{2}(\tau)-\lambda \xi_{3}(\tau)\right] \\
& +\frac{2 \varepsilon}{(2-\varepsilon) \kappa(\varepsilon)} \int_{0}^{\tau}\left[\delta \xi_{2}(k)-\lambda \xi_{3}(k)\right] d k .
\end{aligned}
$$

Now, we consider the following kernels

$$
\begin{aligned}
& \eta\left(\tau, \xi_{1}(\tau)\right)=\lambda-\left(\alpha \xi_{2}(\tau)+\lambda\right) \xi_{1}(\tau), \\
& \sigma\left(\tau, \xi_{2}(\tau)\right)=\alpha \xi_{1}(\tau) \xi_{2}(\tau)-(\lambda+\delta) \xi_{2}(\tau), \\
& \omega\left(\tau, \xi_{3}(\tau)\right)=\delta \xi_{2}(\tau)-\lambda \xi_{3}(\tau) .
\end{aligned}
$$

Theorem 3.1. The kernels $\eta, \sigma$ and $\omega$ hold the Lipschitz condition (LC).

Proof. We consider the mentioned condition for each kernel proposed. Let us take the functions $\xi_{1}$ and $\xi_{4}$, for the kernel $\eta, \xi_{2}$ and $\xi_{5}$, for the kernel $\sigma$, and $\xi_{3}$ and $\xi_{6}$, for the kernel $\omega$. Then we obtain:

$$
\begin{aligned}
& \left\|\eta\left(\tau, \xi_{1}(\tau)\right)-\eta\left(\tau, \xi_{4}(\tau)\right)\right\|=\left\|\left(\alpha \xi_{2}(\tau)+\lambda\right)\left(\xi_{1}(\tau)-\xi_{4}(\tau)\right)\right\|, \\
& \left\|\sigma\left(\tau, \xi_{2}(\tau)\right)-\sigma\left(\tau, \xi_{5}(\tau)\right)\right\|=\left\|\alpha \xi_{1}(\tau)\left(\xi_{2}(\tau)-\xi_{5}(\tau)\right)-(\mu+\gamma)\left(\xi_{2}(\tau)-\xi_{5}(\tau)\right)\right\|, \\
& \left\|\omega\left(\tau, \xi_{3}(\tau)\right)-\omega\left(\tau, \xi_{6}(\tau)\right)\right\|=\left\|\lambda\left(\xi_{3}(\tau)-\xi_{6}(\tau)\right)\right\| .
\end{aligned}
$$

Using Cauchy's inequality in Eq. 3.7, we have

$$
\begin{aligned}
& \left\|\eta\left(\tau, \xi_{1}(\tau)\right)-\eta\left(\tau, \xi_{4}(\tau)\right)\right\| \leq\left\|\left(\alpha \xi_{2}(\tau)+\lambda\right)\left(\xi_{1}(\tau)-\xi_{4}(\tau)\right)\right\|, \\
& \left\|\sigma\left(\tau, \xi_{2}(\tau)\right)-\sigma\left(\tau, \xi_{5}(\tau)\right)\right\| \leq\left\|\alpha \xi_{1}(\tau)\left(\xi_{2}(\tau)-\xi_{5}(\tau)\right)-(\mu+\gamma)\left(\xi_{2}(\tau)-\xi_{5}(\tau)\right)\right\|, \\
& \left\|\omega\left(\tau, \xi_{3}(\tau)\right)-\omega\left(\tau, \xi_{6}(\tau)\right)\right\| \leq\left\|\lambda\left(\xi_{3}(\tau)-\xi_{6}(\tau)\right)\right\| .
\end{aligned}
$$

Considering the following recursive formula, we get

$$
\begin{aligned}
& \xi_{1}(\tau)=\frac{2(1-\varepsilon)}{(2-\varepsilon) \kappa(\varepsilon)} \eta\left(\tau, \xi_{1(\mu-1)}(\tau)\right)+\frac{2 \varepsilon}{(2-\varepsilon) \kappa(\varepsilon)} \int_{0}^{\tau} \eta\left(k, \xi_{1(\mu-1)}(k)\right) d k, \\
& \xi_{2}(\tau)=\frac{2(1-\varepsilon)}{(2-\varepsilon) \kappa(\varepsilon)} \sigma\left(\tau, \xi_{2(\mu-1)}(\tau)\right)+\frac{2 \varepsilon}{(2-\varepsilon) \kappa(\varepsilon)} \int_{0}^{\tau} \sigma\left(k, \xi_{2(\mu-1)}\right) d k, \\
& \xi_{3}(\tau)=\frac{2(1-\varepsilon)}{(2-\varepsilon) \kappa(\varepsilon)} \omega\left(\tau, \xi_{3(\mu-1)}(\tau)\right)+\frac{2 \varepsilon}{(2-\varepsilon) \kappa(\varepsilon)} \int_{0}^{\tau} \omega\left(k, \xi_{3(\mu-1)}\right) d k .
\end{aligned}
$$


Then, we consider the norm and triangular inequality in order to state the difference between the consecutive terms, applying, we can write

$$
\begin{aligned}
\left\|\Upsilon_{\mu}(\tau)\right\|= & \left\|\xi_{1(\mu)}(\tau)-\xi_{4(\mu-1)}(\tau)\right\| \leq \frac{2(1-\varepsilon)}{(2-\varepsilon) \kappa(\varepsilon)}\left\|\eta\left(\tau, \xi_{1(\mu-1)}(\tau)\right)-\eta\left(\tau, \xi_{4(\mu-2)}(\tau)\right)\right\| \\
& +\frac{2 \varepsilon}{(2-\varepsilon) \kappa(\varepsilon)}\left\|\int_{0}^{\tau}\left[\eta\left(k, \xi_{1(\mu-1)}(k)\right)-\eta\left(k, \xi_{4(\mu-2)}(k)\right)\right] d k\right\|, \\
\left\|\Omega_{\mu}(\tau)\right\|= & \left\|\xi_{2(\mu)}(\tau)-\xi_{5(\mu-1)}(\tau)\right\| \leq \frac{2(1-\varepsilon)}{(2-\varepsilon) \kappa(\varepsilon)}\left\|\sigma\left(\tau, \xi_{2(\mu-1)}(\tau)\right)-\sigma\left(\tau, \xi_{5(\mu-2)}(\tau)\right)\right\| \\
& +\frac{2 \varepsilon}{(2-\varepsilon) \kappa(\varepsilon)}\left\|\int_{0}^{\tau}\left[\sigma\left(k, \xi_{2(\mu-1)}(k)\right)-\sigma\left(k, \xi_{5(\mu-2)}(k)\right)\right] d k\right\|, \\
\left\|\Psi_{\mu}(\tau)\right\|= & \left\|\xi_{3(\mu)}(\tau)-\xi_{6(\mu-1)}(\tau)\right\| \leq \frac{2(1-\varepsilon)}{(2-\varepsilon) \kappa(\varepsilon)}\left\|\omega\left(\tau, \xi_{3(\mu-1)}(\tau)\right)-\omega\left(\tau, \xi_{6(\mu-2)}(\tau)\right)\right\| \\
& +\frac{2 \varepsilon}{(2-\varepsilon) \kappa(\varepsilon)}\left\|\int_{0}^{\tau}\left[\omega\left(k, \xi_{3(\mu-1)}(k)\right)-\omega\left(k, \xi_{6(\mu-2)}(k)\right)\right] d k\right\|,
\end{aligned}
$$

where

$$
\xi_{1(\mu)}(\tau)=\sum_{\gamma=0}^{\infty} \Upsilon_{\gamma}(\tau) ; \quad \xi_{2(\mu)}(\tau)=\sum_{\gamma=0}^{\infty} \Omega_{\gamma}(\tau) ; \quad \xi_{3(\mu)}(\tau)=\sum_{\gamma=0}^{\infty} \Psi_{\gamma}(\tau) .
$$

So, the kernels $\eta, \sigma$ and $\omega$ hold the Lipschitz condition, we can write

$$
\begin{aligned}
\left\|\Upsilon_{\mu}(\tau)\right\|= & \left\|\xi_{1(\mu)}(\tau)-\xi_{4(\mu-1)}(\tau)\right\| \leq \frac{2(1-\varepsilon)}{(2-\varepsilon) \kappa(\varepsilon)} \Delta_{1}\left\|\xi_{1(\mu-1)}(\tau)-\xi_{4(\mu-2)}(\tau)\right\| \\
& +\frac{2 \varepsilon}{(2-\varepsilon) \kappa(\varepsilon)} \Delta_{2} \int_{0}^{\tau}\left\|\xi_{1(\mu-1)}(k)-\xi_{4(\mu-2)}(k)\right\| d k, \\
\left\|\Omega_{\mu}(\tau)\right\|= & \left\|\xi_{2(\mu)}(\tau)-\xi_{5(\mu-1)}(\tau)\right\| \leq \frac{2(1-\varepsilon)}{(2-\varepsilon) \kappa(\varepsilon)} \Delta_{3}\left\|\xi_{2(\mu-1)}(\tau)-\xi_{5(\mu-2)}(\tau)\right\| \\
& +\frac{2 \varepsilon}{(2-\varepsilon) \kappa(\varepsilon)} \Delta_{4} \int_{0}^{\tau}\left\|\xi_{2(\mu-1)}(k)-\xi_{5(\mu-2)}(k)\right\| d k, \\
\left\|\Psi_{\mu}(\tau)\right\|= & \left\|\xi_{3(\mu)}(\tau)-\xi_{6(\mu-1)}(\tau)\right\| \leq \frac{2(1-\varepsilon)}{(2-\varepsilon) \kappa(\varepsilon)} \Delta_{5}\left\|\xi_{3(\mu-1)}(\tau)-\xi_{6(\mu-2)}(\tau)\right\| \\
& +\frac{2 \varepsilon}{(2-\varepsilon) \kappa(\varepsilon)} \Delta_{6} \int_{0}^{\tau}\left\|\xi_{1(\mu-1)}(k)-\xi_{4(\mu-2)}(k)\right\| d k .
\end{aligned}
$$

These results complete the proof of Theorem 3.1.

Theorem 3.2. [Existence of the Solution] The system presented by Eq. 3.2 has a solution.

Proof. We show that the kernels $\eta, \sigma$ and $\omega$ satisfy the LC by regarding that Eq. 3.12 is bounded. Taking into consideration the results get in Eq. 3.12 and using the recursive method, we have the following inequality coupled with the relation:

$$
\begin{aligned}
& \left\|\Upsilon_{\mu}(\tau)\right\| \leq\left\|\xi_{1}(0)\right\|+\left\{\left\{\frac{2(1-\varepsilon)}{(2-\varepsilon) \kappa(\varepsilon)} \Delta_{1}\right\}^{\mu}+\left\{\frac{2 \varepsilon}{(2-\varepsilon) \kappa(\varepsilon)} \Delta_{2} \tau\right\}^{\mu}\right\} \\
& \left\|\Omega_{\mu}(\tau)\right\| \leq\left\|\xi_{2}(0)\right\|+\left\{\left\{\frac{2(1-\varepsilon)}{(2-\varepsilon) \kappa(\varepsilon)} \Delta_{3}\right\}^{\mu}+\left\{\frac{2 \varepsilon}{(2-\varepsilon) \kappa(\varepsilon)} \Delta_{4} \tau\right\}^{\mu}{ }^{\mu}\right\} \\
& \left\|\Psi_{\mu}(\tau)\right\| \leq\left\|\xi_{3}(0)\right\|+\left\{\left\{\frac{2(1-\varepsilon)}{(2-\varepsilon) \kappa(\varepsilon)} \Delta_{5}\right\}^{\mu}+\left\{\frac{2 \varepsilon}{(2-\varepsilon) \kappa(\varepsilon)} \Delta_{6} \tau\right\}^{\mu}\right\} .
\end{aligned}
$$

Therefore, Eq. 3.13 exists and is smooth. Nevertheless, we suppose the followings to prove that the functions stated in Eq. 3.13 are a system of solutions of Eq. 3.2,

$$
\begin{aligned}
& \xi_{1}(\tau)=\xi_{1(n)}(\tau)-\Re_{1(n)}(\tau), \\
& \xi_{2}(\tau)=\xi_{2(n)}(\tau)-\Re_{2(n)}(\tau), \\
& \xi_{3}(\tau)=\xi_{3(n)}(\tau)-\Re_{3(n)}(\tau),
\end{aligned}
$$

where $\Re_{1(n)}(\tau), \Re_{2(n)}(\tau)$ and $\Re_{3(n)}(\tau)$ are reminder terms of series solution. Thus,

$$
\begin{aligned}
\xi_{1}(\tau)-\xi_{1(n)}(\tau) & =\frac{2(1-\varepsilon)}{(2-\varepsilon) \kappa(\varepsilon)} \eta\left(\tau, \xi_{1}(\tau)-\Re_{1(n)}(\tau)\right) \\
& +\frac{2 \varepsilon}{(2-\varepsilon) \kappa(\varepsilon)} \int_{0}^{\tau} \eta\left(k, \xi_{1}(\tau)-\Re_{1(n)}(k)\right) d k, \\
\xi_{2}(\tau)-\xi_{2(n)}(\tau) & =\frac{2(1-\varepsilon)}{(2-\varepsilon) \kappa(\varepsilon)} \sigma\left(\tau, \xi_{2}(\tau)-\Re_{2(n)}(\tau)\right) \\
& +\frac{2 \varepsilon}{(2-\varepsilon) \kappa(\varepsilon)} \int_{0}^{\tau} \sigma\left(k, \xi_{2}(\tau)-\Re_{2(n)}(k)\right) d k, \\
\xi_{3}(\tau)-\xi_{3(n)}(\tau) & =\frac{2(1-\varepsilon)}{(2-\varepsilon) \kappa(\varepsilon)} \omega\left(\tau, \xi_{3}(\tau)-\Re_{3(n)}(\tau)\right) \\
& +\frac{2 \varepsilon}{(2-\varepsilon) \kappa(\varepsilon)} \int_{0}^{\tau} \omega\left(k, \xi_{3}(\tau)-\Re_{3(n)}(k)\right) d k .
\end{aligned}
$$


If we take the norm of both sides and use the LC, we get

$$
\begin{aligned}
& \left\|\xi_{1}(\tau)-\xi_{1}(0)-\frac{2(1-\varepsilon)}{(2-\varepsilon) \kappa(\varepsilon)} \eta\left(\tau, \xi_{1}(\tau)\right)-\frac{2 \varepsilon}{(2-\varepsilon) \kappa(\varepsilon)} \int_{0}^{\tau} \eta\left(k, \xi_{1}(k)\right) d k\right\| \\
& \leq\left\|\Re_{1(n)}(\tau)\right\|+\left\{\frac{2(1-\varepsilon)}{(2-\varepsilon) \kappa(\varepsilon)} \Delta_{1}+\frac{2 \varepsilon}{(2-\varepsilon) \kappa(\varepsilon)} \Delta_{2} \tau\right\}\left\|\Re_{1(n)}(\tau)\right\|, \\
& \left\|\xi_{2}(\tau)-\xi_{2}(0)-\frac{2(1-\varepsilon)}{(2-\varepsilon) \kappa(\varepsilon)} \sigma\left(\tau, \xi_{2}(\tau)\right)-\frac{2 \varepsilon}{(2-\varepsilon) \kappa(\varepsilon)} \int_{0}^{\tau} \sigma\left(k, \xi_{2}(k)\right) d k\right\| \\
& \leq\left\|\Re_{2(n)}(\tau)\right\|+\left\{\frac{2(1-\varepsilon)}{(2-\varepsilon) \kappa(\varepsilon)} \Delta_{3}+\frac{2 \varepsilon}{(2-\varepsilon) \kappa(\varepsilon)} \Delta_{4} \tau\right\}\left\|\Re_{2(n)}(\tau)\right\|, \\
& \left\|\xi_{3}(\tau)-\xi_{3}(0)-\frac{2(-\varepsilon)}{(2-\varepsilon) \kappa(\varepsilon)} \omega\left(\tau, \xi_{3}(\tau)\right)-\frac{2 \varepsilon}{(2-\varepsilon) \kappa(\varepsilon)} \int_{0}^{\tau} \omega\left(k, \xi_{3}(k)\right) d k\right\| \\
& \leq\left\|\Re_{3(n)}(\tau)\right\|+\left\{\frac{2(1-\varepsilon)}{(2-\varepsilon) \kappa(\varepsilon)} \Delta_{5}+\frac{2 \varepsilon}{(2-\varepsilon) \kappa(\varepsilon)} \Delta_{6} \tau\right\}\left\|\Re_{3(n)}(\tau)\right\| .
\end{aligned}
$$

Considering the limit $\mu \rightarrow \infty$ of Eq. 3.16, we have

$$
\begin{aligned}
& \xi_{1}(\tau)=\xi_{1}(0)+\frac{2(1-\varepsilon)}{(2-\varepsilon) \kappa(\varepsilon)} \eta\left(\tau, \xi_{1}(\tau)\right)+\frac{2 \varepsilon}{(2-\varepsilon) \kappa(\varepsilon)} \int_{0}^{\tau} \eta\left(k, \xi_{1}(k)\right) d k, \\
& \xi_{2}(\tau)=\xi_{2}(0)+\frac{2(1-\varepsilon)}{(2-\varepsilon) \kappa(\varepsilon)} \sigma\left(\tau, \xi_{2}(\tau)\right)+\frac{2 \varepsilon}{(2-\varepsilon) \kappa(\varepsilon)} \int_{0}^{\tau} \sigma\left(k, \xi_{2}(\tau)\right) d k, \\
& \xi_{3}(\tau)=\xi_{3}(0)+\frac{2(1-\varepsilon)}{(2-\varepsilon) \kappa(\varepsilon)} \omega\left(\tau, \xi_{3}(\tau)\right)+\frac{2 \varepsilon}{(2-\varepsilon) \kappa(\varepsilon)} \int_{0}^{\tau} \omega\left(k, \xi_{3}(\tau)\right) d k .
\end{aligned}
$$

Eq. 3.17 is the solution of Eq. 3.2; accordingly, we conclude that a solution of the system exists.

Theorem 3.3. [Uniqueness of the Solution] The system given by Eq. 3.2 has a unique solution.

Proof. Let us consider $\xi_{1}^{*}(\tau), \xi_{2}^{*}(\tau)$ and $\xi_{3}^{*}(\tau)$, as other solutions for the system 3.2 to show the uniqueness of the solution, then we can write

$$
\begin{aligned}
\xi_{1}(\tau)-\xi_{1}^{*}(\tau)= & \frac{2(1-\varepsilon)}{(2-\varepsilon) \kappa(\varepsilon)}\left[\eta\left(\tau, \xi_{1}(\tau)\right)-\eta\left(\tau, \xi_{1}^{*}(\tau)\right)\right] \\
& +\frac{2 \varepsilon}{(2-\varepsilon) \kappa(\varepsilon)} \int_{0}^{\tau}\left[\eta\left(k, \xi_{1}(k)\right)-\eta\left(k, \xi_{1}^{*}(k)\right)\right] d k, \\
\xi_{2}(\tau)-\xi_{2}^{*}(\tau)= & \frac{2(1-\varepsilon)}{(2-\varepsilon) \kappa(\varepsilon)}\left[\sigma\left(\tau, \xi_{2}(\tau)\right)-\sigma\left(\tau, \xi_{2}^{*}(\tau)\right)\right] \\
& +\frac{2 \varepsilon}{(2-\varepsilon) \kappa(\varepsilon)} \int_{0}^{\tau}\left[\sigma\left(k, \xi_{2}(k)\right)-\sigma\left(k, \xi_{2}^{*}(k)\right)\right] d k, \\
\xi_{3}(\tau)-\xi_{3}^{*}(\tau)= & \frac{2(1-\varepsilon)}{(2-\varepsilon) \kappa(\varepsilon)}\left[\omega\left(\tau, \xi_{3}(\tau)\right)-\omega\left(\tau, \xi_{3}^{*}(\tau)\right)\right] \\
& +\frac{2 \varepsilon}{(2-\varepsilon) \kappa(\varepsilon)} \int_{0}^{\tau}\left[\omega\left(k, \xi_{3}(k)\right)-\omega\left(k, \xi_{3}^{*}(k)\right)\right] d k .
\end{aligned}
$$

Applying the norm to both sides of Eq. 3.18, we have

$$
\begin{aligned}
\left\|\xi_{1}(\tau)-\xi_{1}^{*}(\tau)\right\| & \leq \frac{2(1-\varepsilon)}{(2-\varepsilon) \kappa(\varepsilon)}\left[\left\|\eta\left(\tau, \xi_{1}(\tau)\right)-\eta\left(\tau, \xi_{1}^{*}(\tau)\right)\right\|\right] \\
& +\frac{2 \varepsilon}{(2-\varepsilon) \kappa(\varepsilon)} \int_{0}^{t}\left[\left\|\eta\left(k, \xi_{1}(k)\right)-\eta\left(k, \xi_{1}^{*}(k)\right)\right\|\right] d k, \\
\left\|\xi_{2}(\tau)-\xi_{2}^{*}(\tau)\right\| & \leq \frac{2(-\varepsilon)}{(2-\varepsilon) \kappa(\varepsilon)}\left[\left\|\sigma\left(\tau, \xi_{2}(\tau)\right)-\sigma\left(\tau, \xi_{2}^{*}(\tau)\right)\right\|\right] \\
& +\frac{2 \varepsilon}{(2-\varepsilon) \kappa(\varepsilon)} \int_{0}^{t}\left[\left\|\sigma\left(k, \xi_{2}(k)\right)-\sigma\left(k, \xi_{2}^{*}(k)\right)\right\|\right] d k, \\
\left\|\xi_{3}(\tau)-\xi_{3}^{*}(\tau)\right\| & \leq \frac{2(1-\varepsilon)}{(2-\varepsilon) \kappa(\varepsilon)}\left[\left\|\omega\left(\tau, \xi_{3}(\tau)\right)-\omega\left(\tau, \xi_{3}^{*}(\tau)\right)\right\|\right] \\
& +\frac{2 \varepsilon}{(2-\varepsilon) \kappa(\varepsilon)} \int_{0}^{t}\left[\left\|\omega\left(k, \xi_{3}(k)\right)-\omega\left(k, \xi_{3}^{*}(k)\right)\right\|\right] d k .
\end{aligned}
$$

Taking into account the LC and the solution is bounded, we have

$$
\begin{aligned}
& \left\|\xi_{1}(\tau)-\xi_{1}^{*}(\tau)\right\| \leq \frac{2(1-\varepsilon)}{(2-\varepsilon) \kappa(\varepsilon)} \Delta_{1} \Sigma_{1}+\left\{\frac{2 \varepsilon}{(2-\varepsilon) \kappa(\varepsilon)} \Delta_{2} \Sigma_{2} \tau\right\}^{\mu}, \\
& \left\|\xi_{2}(\tau)-\xi_{2}^{*}(\tau)\right\| \leq \frac{2(1-\varepsilon)}{(2-\varepsilon) \kappa(\varepsilon)} \Delta_{3} \Sigma_{3}+\left\{\frac{2 \varepsilon}{(2-\varepsilon) \kappa(\varepsilon)} \Delta_{4} \Sigma_{4} \tau\right\}^{\mu}, \\
& \left\|\xi_{3}(\tau)-\xi_{3}^{*}(\tau)\right\| \leq \frac{2(1-\varepsilon)}{(2-\varepsilon) \kappa(\varepsilon)} \Delta_{5} \Sigma_{5}+\left\{\frac{2 \varepsilon}{(2-\varepsilon) \kappa(\varepsilon)} \Delta_{6} \Sigma_{6} \tau\right\}^{\mu} .
\end{aligned}
$$

Eq. 3.20 holds for any $\mu$, hence we can obtain

$$
\xi_{1}(\tau)=\xi_{1}^{*}(\tau), \quad \xi_{2}(\tau)=\xi_{2}^{*}(\tau), \quad \xi_{3}(\tau)=\xi_{3}^{*}(\tau) .
$$

This result means that the solution of Eq. 3.2 is unique. 
In this part of the study, we get the solution of the system given by Eq. 3.2 using the LT method. Taking the LT of both sides of Eq. 3.2, we have the following system

$$
\begin{aligned}
& \frac{\ell \mathcal{L}\left\{\xi_{1}(\tau)\right\}-\xi_{1}(0)}{\ell+\varepsilon(1-\ell)}=\mathcal{L}\left\{\lambda-\left(\alpha \xi_{2}(\tau)+\lambda\right) \xi_{1}(\tau)\right\}(\ell), \\
& \frac{\ell \mathcal{L}\left\{\xi_{2}(\tau)\right\}-\xi_{2}(0)}{\ell+\varepsilon(1-\ell)}=\mathcal{L}\left\{\alpha \xi_{1}(\tau) \xi_{2}(\tau)-(\lambda+\delta) \xi_{2}(\tau)\right\}(\ell), \\
& \frac{\ell \mathcal{L}\left\{\xi_{3}(\tau)\right\}-\xi_{3}(0)}{\ell+\varepsilon(1-\ell)}=\mathcal{L}\left\{\delta \xi_{2}(\tau)-\lambda \xi_{3}(\tau)\right\}(\ell) .
\end{aligned}
$$

By applying the inverse LT of the last equation, we obtain

$$
\begin{aligned}
& \xi_{1}(\tau)=\xi_{1}(0)+\mathcal{L}^{-1}\left\{\frac{\ell+\varepsilon(1-\ell)}{\ell} \mathcal{L}\left\{\lambda-\left(\alpha \xi_{2}(\tau)+\lambda\right) \xi_{1}(\tau)\right\}(\ell)\right\}(\tau), \\
& \xi_{2}(\tau)=\xi_{2}(0)+\mathcal{L}^{-1}\left\{\frac{\ell+\varepsilon(1-\ell)}{\ell} \mathcal{L}\left\{\alpha \xi_{1}(\tau) \xi_{2}(\tau)-(\lambda+\delta) \xi_{2}(\tau)\right\}(\ell)\right\}(\tau), \\
& \xi_{3}(\tau)=\xi_{3}(0)+\mathcal{L}^{-1}\left\{\frac{\ell+\varepsilon(1-\ell)}{\ell} \mathcal{L}\left\{\delta \xi_{2}(\tau)-\lambda \xi_{3}(\tau)\right\}(\ell)\right\}(\tau) .
\end{aligned}
$$

Then we get the following recurrence relations

$$
\begin{aligned}
& \xi_{1(\mu)}(\tau)=\mathcal{L}^{-1}\left\{\frac{\ell+\varepsilon(1-\ell)}{\ell} \mathcal{L}\left\{\lambda-\left(\alpha \xi_{2(\mu-1)}(\tau)+\lambda\right) \xi_{1(\mu-1)}(\tau)\right\}(\ell)\right\}(\tau), \\
& \xi_{2(\mu)}(\tau)=\mathcal{L}^{-1}\left\{\frac{\ell+\varepsilon(1-\ell)}{\ell} \mathcal{L}\left\{\alpha \xi_{1(\mu-1)}(\tau) \xi_{2(\mu-1)}(\tau)-(\lambda+\delta) \xi_{2(\mu-1)}(\tau)\right\}(\ell)\right\}(\tau), \\
& \xi_{3(\mu)}(\tau)=\mathcal{L}^{-1}\left\{\frac{\ell+\varepsilon(1-\ell)}{\ell} \mathcal{L}\left\{\delta \xi_{2(\mu-1)}(\tau)-\lambda \xi_{3(\mu-1)}(\tau)\right\}(\ell)\right\}(\tau) .
\end{aligned}
$$

where $\xi_{1(0)}(\tau)=\xi_{1}(0) ; \xi_{2(0)}(\tau)=\xi_{2}(0) ; \xi_{3(0)}(\tau)=\xi_{3}(0)$.

Therefore the approximate solution is presented as a limit when $\mu \rightarrow \infty$

$$
\xi_{1}(\tau)=\lim _{\mu \rightarrow \infty} \xi_{1(\mu)}(\tau) ; \xi_{2}(\tau)=\lim _{\mu \rightarrow \infty} \xi_{2(\mu)}(\tau) ; \xi_{3}(\tau)=\lim _{\mu \rightarrow \infty} \xi_{3(\mu)}(\tau)
$$

Example 1. We give an example to simulate the numerical findings obtained with solutions of the mentioned epidemic model coupled with the $\mathrm{CF}$ fractional operator. We start the simulations with the special initial conditions, $\xi_{1}(0)=20, \xi_{2}(0)=1, \xi_{3}(0)=1$, and $\lambda=0.1, \alpha=0.5, \delta=0.1$.

The simulations results presented by the following figures demonstrate that the numerical computations with respect to the special solutions of the suggested model are very efficient and accurate.

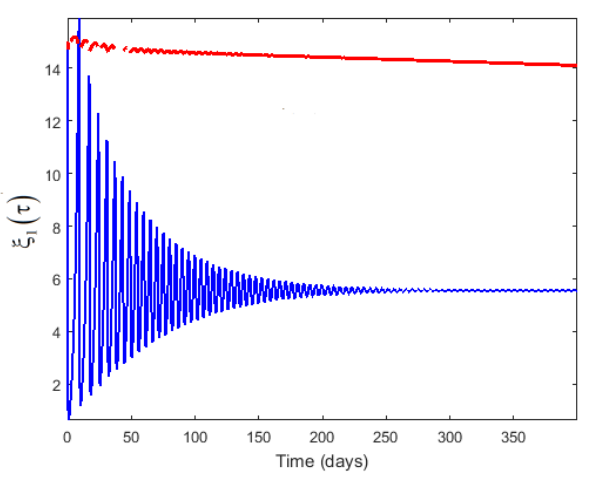

Figure 1. The numerical results of susceptible individuals $\left(\xi_{1}\right)$ in the CF operator mean when $\varepsilon=0.5$. 


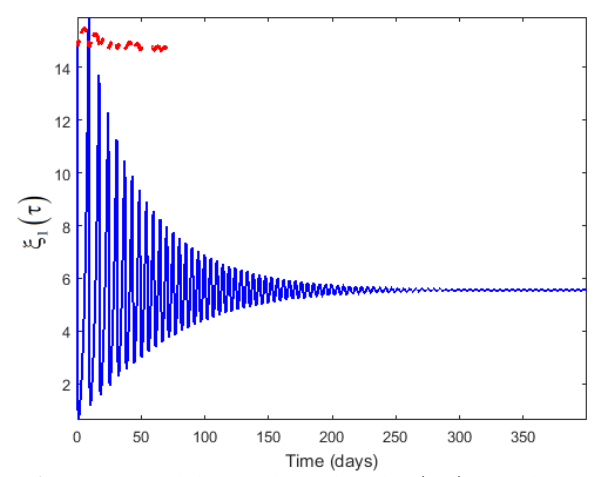

Figure 2. The numerical results of susceptible individuals $\left(\xi_{1}\right)$ in the CF operator mean when $\varepsilon=0.7$.

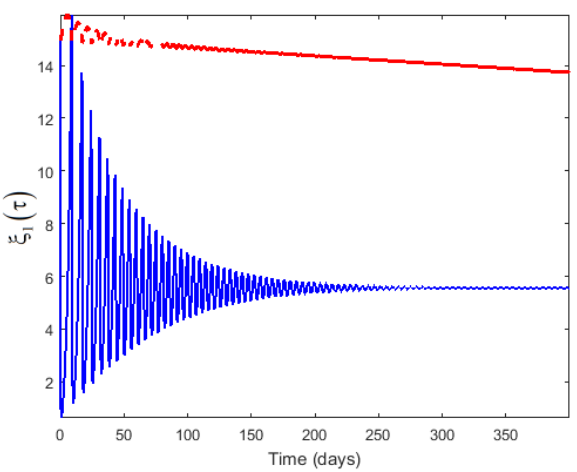

Figure 3. The numerical results of susceptible individuals $\left(\xi_{1}\right)$ in the $\mathrm{CF}$ operator mean when $\varepsilon=1.0$.

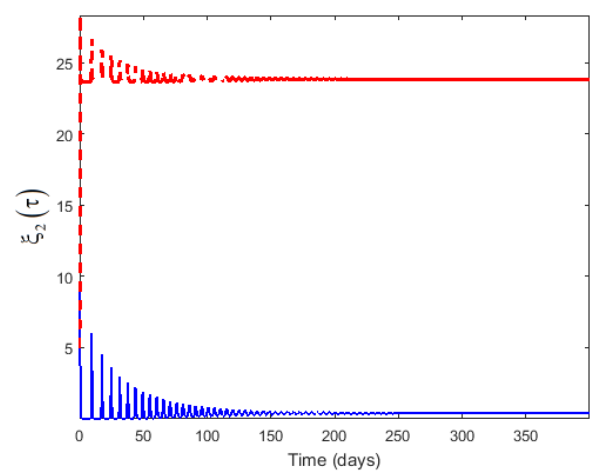

Figure 4. The numerical results of infected individuals $\left(\xi_{2}\right)$ in the CF operator mean when $\varepsilon=0.5$.

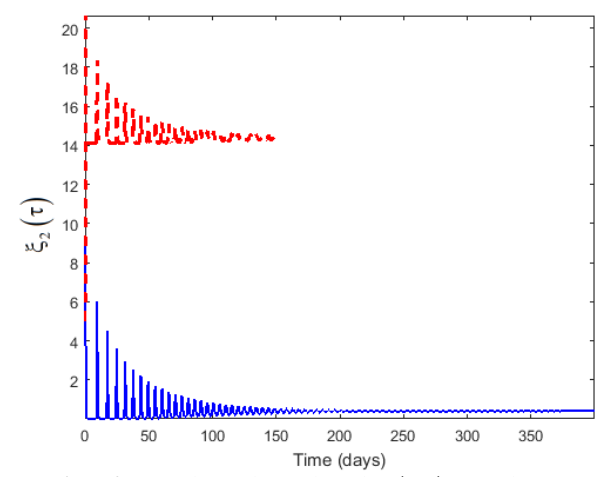

Figure 5. The numerical results of infected individuals $\left(\xi_{2}\right)$ in the CF operator mean when $\varepsilon=0.7$. 


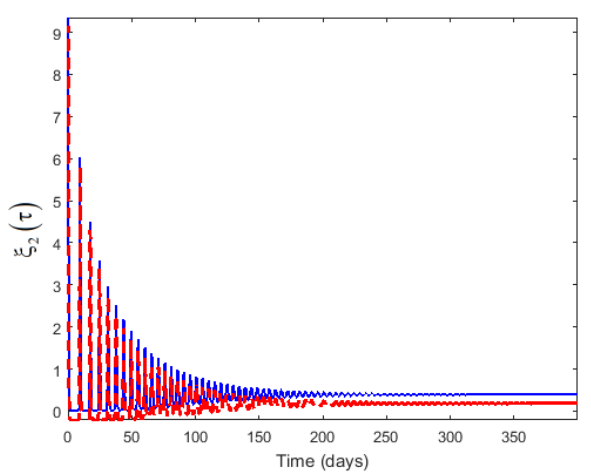

Figure 6. The numerical results of infected individuals $\left(\xi_{2}\right)$ in the CF operator mean when $\varepsilon=1.0$.

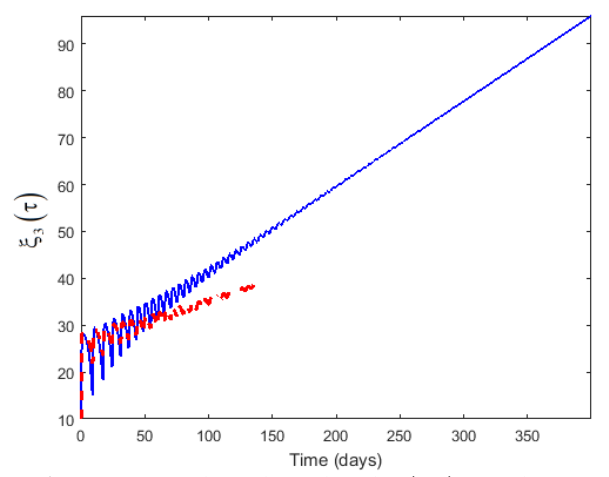

Figure 7. The numerical results of recovered individuals $\left(\xi_{3}\right)$ in the CF operator mean when $\varepsilon=0.5$.

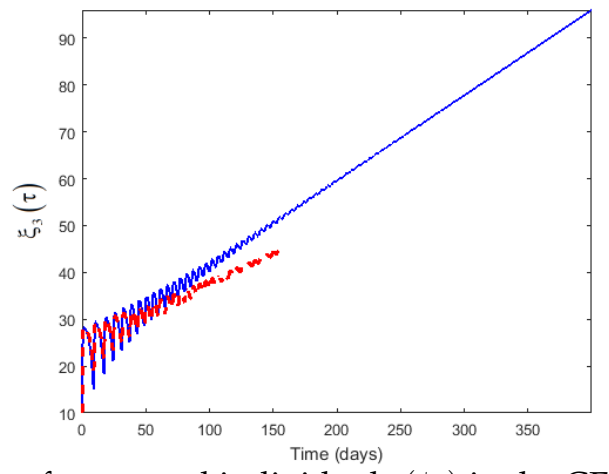

Figure 8. The numerical results of recovered individuals $\left(\xi_{3}\right)$ in the CF operator mean when $\varepsilon=0.7$.

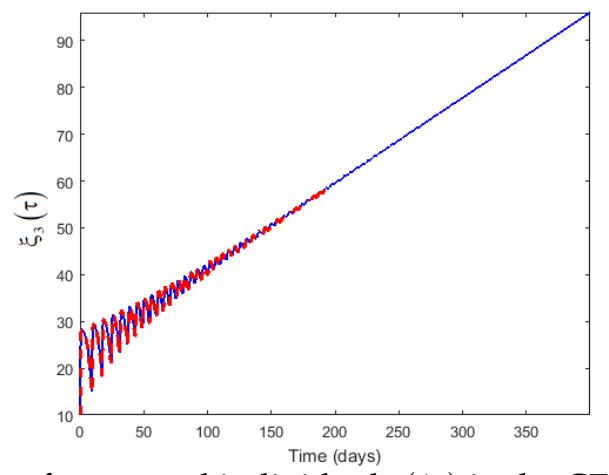

Figure 9. The numerical results of recovered individuals $\left(\xi_{3}\right)$ in the $\mathrm{CF}$ operator mean when $\varepsilon=1.0$. 


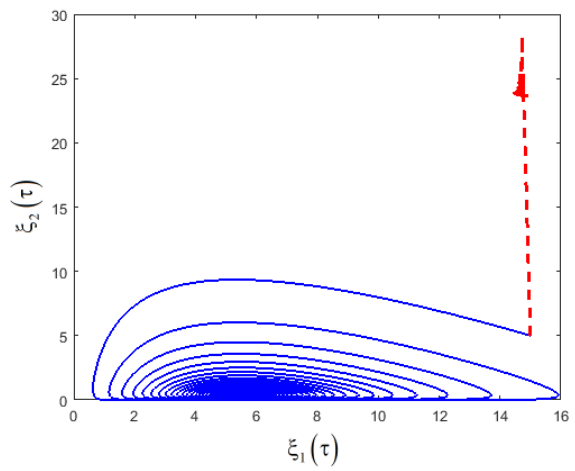

Figure 10. The numerical simulations projected onto $\xi_{1}(\tau)-\xi_{2}(\tau)$ in the CF operator mean when $\varepsilon=0.5$.

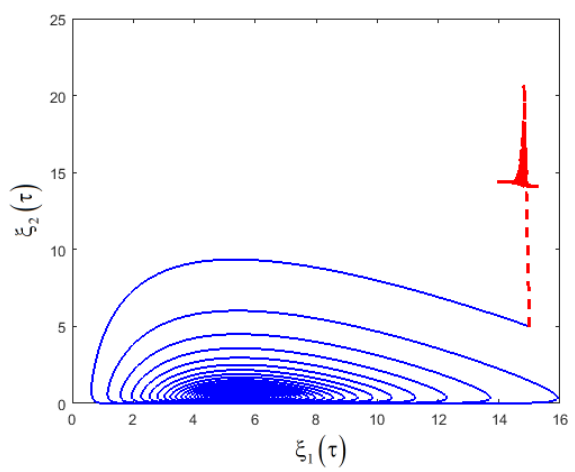

Figure 11. The numerical simulations projected onto $\xi_{1}(\tau)-\xi_{2}(\tau)$ in the CF operator mean when $\varepsilon=0.7$.

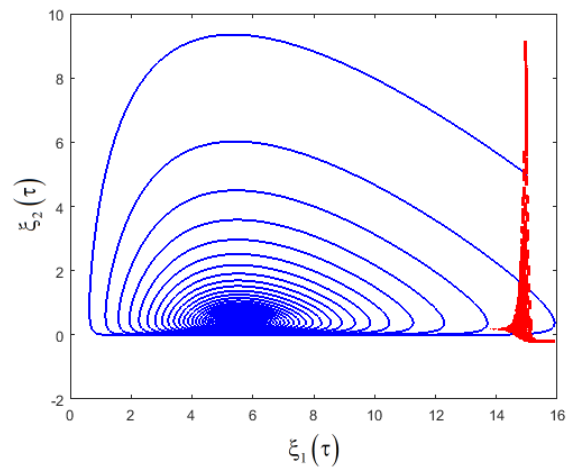

Figure 12. The numerical simulations projected onto $\xi_{1}(\tau)-\xi_{2}(\tau)$ in the CF operator mean when $\varepsilon=1.0$.

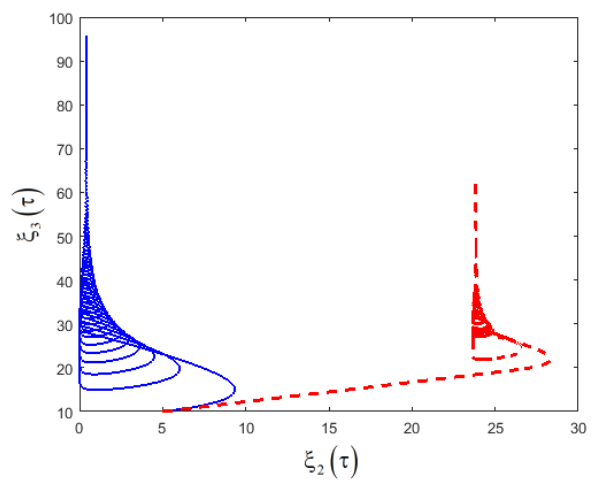

Figure 13. The numerical simulations projected onto $\xi_{2}(\tau)-\xi_{3}(\tau)$ in the CF operator mean when $\varepsilon=0.5$. 


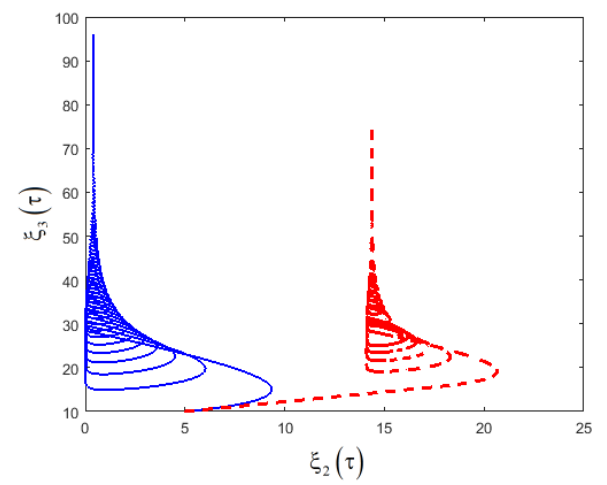

Figure 14. The numerical simulations projected onto $\xi_{2}(\tau)-\xi_{3}(\tau)$ in the CF operator mean when $\varepsilon=0.7$.

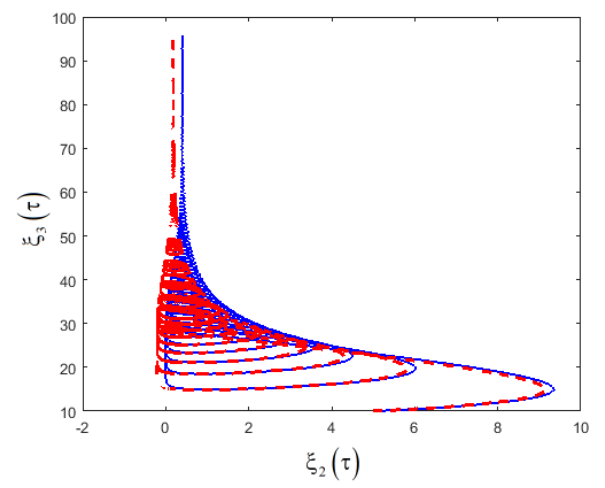

Figure 15. The numerical simulations projected onto $\xi_{2}(\tau)-\xi_{3}(\tau)$ in the CF operator mean when $\varepsilon=1.0$.

\section{Concluding Remarks}

In this paper, we have analyzed a three-dimensional fractional dynamical SIR epidemic model. This model categorizes individuals in a population as Susceptible (S), Infected (I) and Recovered (R). It also simulates the transmission dynamics of diseases where individuals acquire permanent immunity. The SIR model has been considered via the Caputo-Fabrizio fractional derivative. The solutions of the alternative models were obtained using an iterative scheme for the CFC fractional operator based on the Laplace transform. Furthermore, the stability analysis of the iterative method and the uniqueness of the special solutions have been presented with figures. The results we obtained have shown that the concept of fractional differentiation is a powerful mathematical tool to express the non-locality of a given dynamical system. This is because the operator has crossover property which makes it possible to capture complex phenomena and gives an accurate prediction.

\section{Acknowledgements}

M. Yavuz was supported by TUBITAK (The Scientific and Technological Research Council of Turkey).

\section{References}

[1] De León, C. V.: Constructions of Lyapunov Functions for Classics SIS, SIR and SIRS Epidemic Model with Variable Population Size. Foro-Red-Mat: Revista Electrónica de Contenido Matemático. 26, 1-12 (2009).

[2] Kermark, M., Mckendrick, A.: Contributions to the Mathematical Theory of Epidemics. Part I. Proc. R. Soc. A. 115, 700-721 (1927).

[3] Arqub, O. A., El-Ajou, A.: Solution of the Fractional Epidemic Model by Homotopy Analysis Method. Journal of King Saud University-Science. 25 (1), 73-81 (2013).

[4] El-Saka, H.: The Fractional-Order SIR and SIRS Epidemic Models with Variable Population Size. Mathematical Sciences Letters. 2 (3), 195 (2013). 
[5] Angstmann, C. N., Henry, B. I., McGann, A. V.: A Fractional-Order Infectivity SIR Model. Physica A: Statistical Mechanics and its Applications. 452, 86-93 (2016).

[6] Arenas, A. J., González-Parra, G., Chen-Charpentier, B. M.: Construction of Nonstandard Finite Difference Schemes for the SI and SIR Epidemic Models of Fractional Order. Mathematics and Computers in Simulation. 121, 48-63 (2016).

[7] Angstmann, C., Henry, B., McGann, A.: A Fractional Order Recovery SIR Model from a Stochastic Process. Bulletin of Mathematical Biology. 78 (3), 468-499 (2016).

[8] Demirci, E., Unal, A., Özalp, N.: A Fractional Order SEIR Model with Density Dependent Death Rate. Hacettepe Journal of Mathematics and Statistics. 40 (2), 287-295 (2011).

[9] Bonyah, E., Atangana, A., Khan, M. A.: Modeling the Spread of Computer Virus Via Caputo Fractional Derivative and the Beta-Derivative. Asia Pacific Journal on Computational Engineering. 4 (1), 1-15 (2017).

[10] Bonyah, E., Gómez-Aguilar, J., Adu, A.: Stability Analysis and Optimal Control of a Fractional Human African Trypanosomiasis Model. Chaos, Solitons and Fractals. 117, 150-160 (2018).

[11] Yavuz, M., Bonyah, E.: New approaches to the fractional dynamics of schistosomiasis disease model. Physica A: Statistical Mechanics and its Applications, 525, 373-393 (2019).

[12] Aguirre-Ramos, H., Avina-Cervantes, J. G., Cruz-Aceves, I., Ruiz-Pinales, J., Ledesma, S.: Blood Vessel Segmentation in Retinal Fundus Images Using Gabor Filters, Fractional Derivatives, and Expectation Maximization. Applied Mathematics and Computation. 339, 568-587 (2018).

[13] Prakash, A., Kumar, M.: Numerical Solution of Two Dimensional Time Fractional-order Biological Population Model. Open Physics. 14, 177-186 (2016).

[14] Elazzouzi, A., Alaoui, A. L., Tilioua, M., Torres, D. F.: Analysis of a SIRI Epidemic Model with Distributed Delay and Relapse. Statistics, Optimization \& Information Computing. 7 (3), 545-557 (2019).

[15] Caputo, M., Fabrizio, M.: A New Definition of Fractional Derivative without Singular Kernel. Progress in Fractional Differentiation and Applications. 1 (2), 1-13 (2015).

[16] Atangana, A., Baleanu, D.: New Fractional Derivatives with Nonlocal and Non-Singular Kernel: Theory and Application to Heat Transfer Model. Thermal Science. 20 (2), 763-769 (2016).

[17] Atangana, A., Koca, I.: Chaos in a Simple Nonlinear System with Atangana-Baleanu Derivatives with Fractional Order. Chaos, Solitons and Fractals. 89, 447-454 (2016).

[18] Yang, X. J., Gao, F., Machado, J. A., Baleanu, D.: A New Fractional Derivative Involving the Normalized Sinc Function without Singular Kernel. The European Physical Journal Special Topics. 226, 3567-3575 (2017).

[19] Algahtani, O. J. J.: Comparing the Atangana-Baleanu and Caputo-Fabrizio Derivative with Fractional Order: Allen Cahn Model. Chaos, Solitons and Fractals. 89, 552-559 (2016).

[20] Atangana, A., Baleanu, D.: Caputo-Fabrizio Derivative Applied to Groundwater Flow within Confined Aquifer. Journal of Engineering Mechanics. 143, D4016005-1-5 (2017).

[21] Baleanu, D., Agheli, B., Al Qurashi, M. M.: Fractional Advection Differential Equation within Caputo and Caputo-Fabrizio Derivatives. Advances in Mechanical Engineering. 8 (12), 1-8 (2016).

[22] Gómez-Aguilar, J. F., Morales-Delgado, V. F., Taneco-Hernández, M. A., Baleanu, D., Escobar-Jiménez, R. F., Al Qurashi, M. M.: Analytical Solutions of the Electrical Rlc Circuit Via Liouville-Caputo Operators with Local and Non-Local Kernels. Entropy. 18, 402 (2016).

[23] Hristov, J.: Transient Heat Diffusion with a Non-Singular Fading Memory: From the Cattaneo Constitutive Equation with Jeffrey's Kernel to the Caputo-Fabrizio Time-Fractional Derivative. Thermal Science. 20 (2), 757-762 (2016).

[24] Hristov, J.: Steady-State Heat Conduction in a Medium with Spatial Non-Singular Fading Memory: Derivation of Caputo-Fabrizio Space-Fractional Derivative from Cattaneo Concept with Jeffrey's Kernel and Analytical Solutions. Thermal science. 21 (2), 827-839 (2017). 
[25] Sheikh, N. A., Ali, F., Saqib, M., Khan, I., Jan, S. A. A., Alshomrani, A. S., Alghamdi, M. S.: Comparison and Analysis of the Atangana-Baleanu and Caputo-Fabrizio Fractional Derivatives for Generalized Casson Fluid Model with Heat Generation and Chemical Reaction. Results in Physics. 7, 789-800 (2017).

[26] Yavuz, M., Özdemir, N.: European Vanilla Option Pricing Model of Fractional Order without Singular Kernel. Fractal and Fractional. 2 (3), 1-11 (2018).

[27] Evirgen, F., Yavuz, M.: An Alternative Approach for Nonlinear Optimization Problem with Caputo-Fabrizio Derivative, In: Proceedings, ITM Web of Conferences: EDP Sciences, 01009, (2018).

[28] Singh, J., Kumar, D., Hammouch, Z., Atangana, A.: A Fractional Epidemiological Model for Computer Viruses Pertaining to a New Fractional Derivative. Applied Mathematics and Computation. 316, 504-515 (2018).

[29] Yavuz, M. Characterizations of two different fractional operators without singular kernel. Mathematical Modelling of Natural Phenomena, 14 (3), 302 (2019).

[30] Avc1, D., Yetim, A. Analytical Solutions to the Advection-Diffusion Equation with the Atangana-Baleanu Derivative over a Finite Domain. Balıkesir Üniversitesi Fen Bilimleri Enstitüsü Dergisi, 20 (2), 382-395 (2018).

[31] Avc1, D., Yavuz, M., Özdemir, N.: Fundamental solutions to the Cauchy and Dirich-let problems for a heat conduction equation equipped with the Caputo-Fabrizio differentiation. In: Heat Conduction: Methods, Applications and Research. Nova Science Publishers, 95-107 (2019).

[32] Özdemir, N., Uçar, S., Eroğlu, B. B. İ.: Dynamical Analysis of Fractional Order Model for Computer Virus Propagation with Kill Signals. International Journal of Nonlinear Sciences and Numerical Simulation, (2020). https://doi.org/10.1515/ijnsns-2019-0063

[33] Evirgen, F., Uçar, S., Özdemir, N., Hammouch, Z.: System Response of an Alcoholism Model Under the Effect of Immigration via Non-singular Kernel Kerivative. Discrete \& Continuous Dynamical Systems-S, (2020). https://doi.org/10.3934/dcdss.2020145

[34] Ucar, E., Özdemir, N., Altun, E.: Fractional Order Model of Immune Cells Influenced by Cancer Cells. Mathematical Modelling of Natural Phenomena, 14 (3), 308 (2019).

[35] Yavuz, M.: Fundamental Solution of Heat Problem with a New Fractional Derivative Operator Involving Normalized Sinc Function. In: Proceedings of the Mathematical Studies and Applications, 4-6 October 2018, Karaman, TURKEY. Proceedings, 194-198 (2018).

[36] Yavuz, M., Özdemir, N.: An Integral Transform Solution for Fractional Advection-Diffusion Problem. In: Proceedings of the Mathematical Studies and Applications, 4-6 October 2018, Karaman, TURKEY. Proceedings, $442-446$ (2018).

[37] Yavuz, M., Abdeljawad, T: On a Nem Integral Transformation Applied to Fractional Derivative with Mittag-Leffler Nonsingular Kernel. Electronic Research Archive, 28 (1), 1-11 (2020). https:/ /doi.org/10.3934/era.2020039

[38] Acay, B., Bas, E., Abdeljawad, T.: Non-local fractional calculus from different viewpoint generated by truncated M-derivative. Journal of Computational and Applied Mathematics, 366, 112410, (2020).

[39] Bas, E., Ozarslan, R., Baleanu, D., Ercan, A.: Comparative simulations for solutions of fractional Sturm-Liouville problems with non-singular operators. Advances in Difference Equations, 2018 (1), 350 (2018).

[40] Sene, N.: Stability Analysis of the Fractional Differential Equations with the Caputo-Fabrizio Fractional Derivative. Journal of Fractional Calculus and Applications, 11 (2), 160-172 (2020).

[41] Naik, P. A., Zu, J., Ghoreishi, M.: Estimating the approximate analytical solution of HIV viral dynamic model by using homotopy analysis method. Chaos, Solitons \& Fractals, 131, 109500 (2019).

[42] Yavuz, M., Ozdemir, N., Baskonus, H. M.: Solutions of Partial Differential Equations Using the Fractional Operator Involving Mittag-Leffler Kernel. The European Physical Journal Plus. 133, 215 (2018).

[43] Uçar, S., Uçar, E., Ozdemir, N., Hammouch, Z.: Mathematical Analysis and Numerical Simulation for a Smoking Model with Atangana-Baleanu Derivative. Chaos, Solitons and Fractals. 118, 300-306 (2019). 
[44] Yavuz, M.: Novel Recursive Approximation for Fractional Nonlinear Equations within Caputo-Fabrizio Operator. In: Proceedings, ITM Web of Conferences: EDP Sciences, 01008, (2018).

[45] Keten, A., Yavuz, M., Baleanu, D.: Nonlocal Cauchy Problem via a Fractional Operator Involving Power Kernel in Banach Spaces. Fractal and Fractional, 3 (2), 27 (2019).

[46] Sulaiman, T. A., Yavuz, M., Bulut, H., Baskonus, H. M.: Investigation of the fractional coupled viscous Burgers' equation involving Mittag-Leffler kernel. Physica A: Statistical Mechanics and its Applications, 527, 121126, (2019).

[47] Asif, N. A., Hammouch, Z., Riaz, M. B., Bulut, H.: Analytical solution of a Maxwell fluid with slip effects in view of the Caputo-Fabrizio derivative. The European Physical Journal Plus, 133 (7), 272 (2018).

[48] Bas, E., Acay, B., Ozarslan, R.: Fractional models with singular and non-singular kernels for energy efficient buildings. Chaos: An Interdisciplinary Journal of Nonlinear Science, 29 (2), 023110 (2019).

[49] Luchko, Y., Gorenflo, R.: An operational method for solving fractional differential equations with the Caputo derivatives. Acta Mathematica Vietnamica, 24 (2), 207-233 (1999).

[50] Baleanu, D., Diethelm, K., Scalas, E., Trujillo, J. J.: Fractional Calculus: Models and Numerical Methods, World Scientific, (2012).

[51] Miller, K. S., Ross, B.: An Introduction to the Fractional Calculus and Fractional Differential Equations, New York, John Wiley and Sons, (1993).

[52] Atangana, A., Alkahtani, B. S. T.: New Model of Groundwater Flowing within a Confine Aquifer: Application of Caputo-Fabrizio Derivative. Arabian Journal of Geosciences. 9 (8), 1-6 (2016).

[53] Losada, J., Nieto, J. J.: Properties of a New Fractional Derivative without Singular Kernel. Progr. Fract. Differ. Appl. 1 (2), 87-92 (2015).

[54] Shulgin, B., Stone, L., Agur, Z.: Pulse Vaccination Strategy in the Sir Epidemic Model. Bulletin of Mathematical Biology. 60 (6), 1123-1148 (1998).

\section{Affiliations}

\section{MEHMET YAVUZ}

AdDRESS: Necmettin Erbakan University, Dept. of Mathematics-Computer, 42090, Konya-Turkey. University of Exeter, Dept. of Mathematics, TR10, Exeter-UK.

E-MAIL: mehmetyavuz@erbakan.edu.tr, m.yavuz@exeter.ac.uk

ORCID ID: 0000-0002-3966-6518

NECATI ÖZDEMIR

ADDRESS: Balikesir University, Dept. of Mathematics, 10145, Balikesir-Turkey.

E-MAIL: nozdemir@balikesir.edu.tr

ORCID ID: 0000-0002-6339-1868 